\title{
Singular fibers of stable maps of 3-manifolds with boundary into surfaces and their applications
}

\author{
OSAMU SAEKI \\ TAKAHIRO YAMAMOTO
}

\begin{abstract}
We first classify singular fibers of proper $C^{\infty}$ stable maps of 3-dimensional manifolds with boundary into surfaces. Then we compute the cohomology groups of the associated universal complex of singular fibers, and obtain certain cobordism invariants for Morse functions on compact surfaces with boundary.
\end{abstract}

57R45; 57R35, 57R90, 58K15, 58K65

\section{Introduction}

Let $M$ and $N$ be smooth manifolds, where $M$ may possibly have boundary, while $N$ has no boundary. For a $C^{\infty}$ map $f: M \rightarrow N$ and a point $q \in N$, the preimage $f^{-1}(q)$ is called the level set of $f$ over $q$. Throughout this paper, we call the map germ along the level set

$$
f:\left(M, f^{-1}(q)\right) \rightarrow(N, q)
$$

the fiber over $q$, adopting the terminology introduced in Saeki [7]. Furthermore, if a point $q \in N$ is a regular value of both $f$ and $\left.f\right|_{\partial M}$, then we call the fiber (or the level set) over $q$ a regular fiber (resp. a regular level set); otherwise, a singular fiber (resp. a singular level set).

Some equivalence relations among fibers are defined as follows. Let $f_{i}: M_{i} \rightarrow N_{i}$, $i=0,1$, be $C^{\infty}$ maps. For $q_{i} \in N_{i}, i=0,1$, we say that the fibers over $q_{0}$ and $q_{1}$ are $C^{\infty}$ equivalent (or $C^{0}$ equivalent) if, for some open neighborhoods $U_{i}$ of $q_{i}$ in $N_{i}$, there exist diffeomorphisms (resp. homeomorphisms) $\Phi: f_{0}^{-1}\left(U_{0}\right) \rightarrow f_{1}^{-1}\left(U_{1}\right)$ and $\varphi: U_{0} \rightarrow U_{1}$ with $\varphi\left(q_{0}\right)=q_{1}$ which make the following diagram commutative:

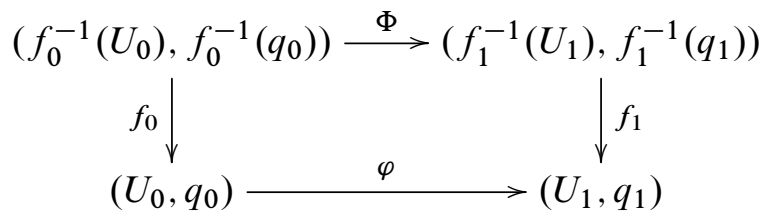


Denote by $C^{\infty}(M, N)$ the set of $C^{\infty}$ maps $M \rightarrow N$ equipped with the Whitney $C^{\infty}$ topology. A $C^{\infty}$ map $f: M \rightarrow N$ is called a $C^{\infty}$ stable map (or a $C^{0}$ stable map) if there exists a neighborhood $N(f) \subset C^{\infty}(M, N)$ of $f$ such that every map $g \in N(f)$ is $C^{\infty}$ right-left equivalent (resp. $C^{0}$ right-left equivalent) to $f$; see Golubitsky and Guillemin [3]. Here, two maps $f$ and $g \in C^{\infty}(M, N)$ are $C^{\infty}$ right-left equivalent (or $C^{0}$ right-left equivalent) if there exist diffeomorphisms (resp. homeomorphisms) $\Psi: M \rightarrow M$ and $\psi: N \rightarrow N$ such that $f \circ \Psi=\psi \circ g$. When we just say that $f$ is a stable map, it will mean that it is a $C^{\infty}$ stable map.

The notion of singular fibers of $C^{\infty}$ maps between manifolds without boundary was first introduced in [7], where classifications of singular fibers of stable maps $M \rightarrow N$ with $(\operatorname{dim} M, \operatorname{dim} N)=(2,1),(3,2)$ and $(4,3)$ were obtained. Later, singular fibers of stable maps of manifolds without boundary were studied in Saeki [7;8], Saeki and Yamamoto $[11 ; 12]$ and Yamamoto $[16 ; 17 ; 18]$, especially in connection with cobordisms. The first author [7] established the theory of universal complex of singular fibers of $C^{\infty}$ maps as an analogy for the Vassiliev complex for map germs; see Ohmoto [5] and Vassiliev [15]. This can be used for getting certain cobordism invariants of singular maps. For example, the first author [7] obtained cobordism invariants for stable Morse functions on closed surfaces, and the second author [18] studied the universal complex of singular fibers of two-colored $C^{\infty}$ maps, computing its cohomology groups. In these theories, for a certain set of singular fibers $\tau$, cohomology classes of the universal complexes of singular fibers of $\tau$-maps provide $\tau$-cobordism invariants for $\tau$-maps.

In this paper, we study singular fibers of proper $C^{\infty}$ stable maps of 3-dimensional manifolds with boundary into surfaces without boundary. By using such fibers, we obtain cobordism invariants for stable Morse functions on compact surfaces with boundary.

The paper is organized as follows. In Section 2, we classify fibers of proper $C^{\infty}$ stable maps of 3-dimensional manifolds with boundary into surfaces without boundary, with respect to the $C^{\infty}$ equivalence relation. For this we use several known results on the classification of stable singularities of maps on manifolds with boundary (see Martins and Nabarro [4] and Shibata [14]) together with the techniques developed by the first author in [7]. In Section 3, we obtain several coexistence formulae of singular fibers of $C^{\infty}$ stable maps of compact 3-dimensional manifolds with boundary into surfaces without boundary. These formulae can be obtained by analyzing the adjacencies of the singular fibers. In Section 4, we construct the universal complex of singular fibers of proper $C^{\infty}$ stable maps of 3-dimensional manifolds with boundary into surfaces without boundary. By carefully computing the cohomology groups of the universal complex, we obtain certain cobordism invariants for stable Morse functions on compact surfaces with boundary. 
Throughout the paper, all manifolds and maps between them are smooth of class $C^{\infty}$ unless otherwise stated. For a smooth map $f: M \rightarrow N$ between manifolds, we denote by $S(f)$ the set of points in $M$ where the differential of $f$ does not have maximal rank $\min \{\operatorname{dim} M, \operatorname{dim} N\}$. For a space $X, \operatorname{id}_{X}$ denotes the identity map of $X$. For a (co)cycle $c$, we denote by $[c]$ the (co)homology class represented by $c$.

\section{Classification of singular fibers}

In this section, we classify singular fibers of proper $C^{\infty}$ stable maps of 3-dimensional manifolds with boundary into surfaces without boundary.

We can prove the following characterization of $C^{\infty}$ stable maps $f: M \rightarrow N$ by using standard techniques in singularity theory together with the results on local normal forms obtained by Martins and Nabarro and by Shibata.

Proposition 2.1 [14; 4] Let $M$ be a 3-manifold, possibly with boundary, and $N$ a surface without boundary. A proper $C^{\infty}$ map $f: M \rightarrow N$ is $C^{\infty}$ stable if and only if it satisfies the following conditions.

(1) Local conditions In the following, for $p \in \partial M$, we use local coordinates $(x, y, z)$ around $p$ such that Int $M$ and $\partial M$ correspond to the sets $\{z>0\}$ and $\{z=0\}$, respectively.

(1a) For $p \in \operatorname{Int} M$, the germ of $f$ at $p$ is right-left equivalent to one of the following:

$$
(x, y, z) \mapsto \begin{cases}(x, y), & p: \text { regular point, } \\ \left(x, y^{2}+z^{2}\right), & p: \text { definite fold point, } \\ \left(x, y^{2}-z^{2}\right), & p: \text { indefinite fold point, } \\ \left(x, y^{3}+x y-z^{2}\right), & p: \text { cusp point. }\end{cases}
$$

(1b) For $p \in \partial M \backslash S(f)$, the germ of $f$ at $p$ is right-left equivalent to one of the following:

$$
(x, y, z) \mapsto \begin{cases}(x, y), & p: \text { regular point of }\left.f\right|_{\partial M}, \\ \left(x, y^{2}+z\right), & p: \text { boundary definite fold point, } \\ \left(x, y^{2}-z\right), & p: \text { boundary indefinite fold point, } \\ \left(x, y^{3}+x y+z\right), & p: \text { boundary cusp point. }\end{cases}
$$

(1c) For $p \in \partial M \cap S(f)$, the germ of $f$ at $p$ is right-left equivalent to the map germ

$$
(x, y, z) \mapsto\left(x, y^{2}+x z \pm z^{2}\right) .
$$




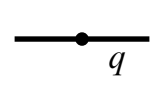

(1)

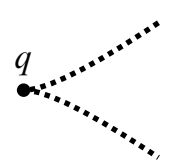

$(5)$

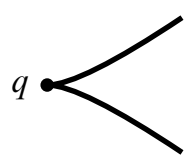

(2)

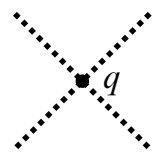

(6)

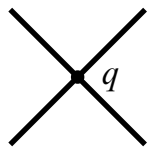

(3)

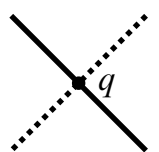

(7)

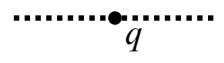

(4)

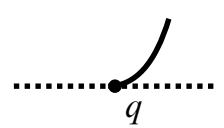

(8)

Figure 1: The images of multigerms of $\left.f\right|_{S(f) \cup S\left(\left.f\right|_{\partial M}\right)}$

(2) Global conditions For each $q \in f(S(f)) \cup f\left(S\left(\left.f\right|_{\partial M}\right)\right)$, the multigerm

$$
\left(\left.f\right|_{S(f) \cup S\left(\left.f\right|_{\partial M}\right)}, f^{-1}(q) \cap\left(S(f) \cup S\left(\left.f\right|_{\partial M}\right)\right)\right)
$$

is right-left equivalent to one of the eight multigerms whose images are depicted in Figure 1, where the ordinary curves correspond to $f(S(f))$ and the dotted curves to $f\left(S\left(\left.f\right|_{\partial M}\right)\right)$ : (1) and (4) represent immersion mono-germs $(\mathbb{R}, 0) \rightarrow\left(\mathbb{R}^{2}, 0\right), t \mapsto(t, 0)$, which correspond to a single fold point and a single boundary fold point, respectively; (3), (6) and (7) represent normal crossings of two immersion germs, each of which corresponds to a fold point or a boundary fold point; (2) and (5) represent cusp mono-germs $(\mathbb{R}, 0) \rightarrow\left(\mathbb{R}^{2}, 0\right)$, $t \mapsto\left(t^{2}, t^{3}\right)$, which correspond to a cusp point and a boundary cusp point, respectively; and (8) represents the restriction of the mono-germ (1c), corresponding to a single point in $\partial M \cap S(f)$, to the singular point set.

Note that if a $C^{\infty}$ map $f: M \rightarrow N$ is $C^{\infty}$ stable, then so is $\left.f\right|_{\partial M}: \partial M \rightarrow N$.

In the following, a point $p$ on the boundary such that the map germ at $p$ is right-left equivalent to the normal form

$$
(x, y, z) \mapsto\left(x, y^{2}+x z+z^{2}\right) \quad \text { or } \quad(x, y, z) \mapsto\left(x, y^{2}+x z-z^{2}\right)
$$

is called a definite $\Sigma_{1,0}^{2,0}$ point or an indefinite $\Sigma_{1,0}^{2,0}$ point, respectively.

Let $M_{i}$ be smooth manifolds and $A_{i} \subset M_{i}$ be subsets, $i=0,1$. A continuous map $g: A_{0} \rightarrow A_{1}$ is said to be smooth if for every $q \in A_{0}$ there exists a smooth map $\tilde{g}: V \rightarrow M_{1}$ defined on a neighborhood $V$ of $q \in M_{0}$ such that $\left.\tilde{g}\right|_{V \cap A_{0}}=$ $\left.g\right|_{V \cap A_{0}}$. Furthermore, a smooth map $g: A_{0} \rightarrow A_{1}$ is called a diffeomorphism if it is a homeomorphism and its inverse is also smooth. When there exists a diffeomorphism between $A_{0}$ and $A_{1}$, we say that they are diffeomorphic. 
Then, by Proposition 2.1, we have the following local descriptions on singular level sets. In the statement, it may not be necessary to distinguish some of the cases if only the topology of level sets is concerned; nevertheless, we divide the cases as below in order to introduce symbols which take corresponding map germs into account.

Lemma 2.2 Let $M$ be a 3-manifold, possibly with boundary, $N$ a surface without boundary, and $f: M \rightarrow N$ a proper $C^{\infty}$ stable map. Then every point $p \in$ $S(f) \cup S\left(\left.f\right|_{\partial M}\right)$ has one of the following neighborhoods in its corresponding singular level set (see Figures 2 and 3 for reference):

(1) Isolated point diffeomorphic to $\left\{(y, z) \in \mathbb{R}^{2} \mid y^{2}+z^{2}=0\right\}$, if $p \in \operatorname{Int} M$ is a definite fold point.

(2) Union of two transverse arcs diffeomorphic to $\left\{(y, z) \in \mathbb{R}^{2} \mid y^{2}-z^{2}=0\right\}$, if $p \in \operatorname{Int} M$ is an indefinite fold point.

(3) Cuspidal arc diffeomorphic to $\left\{(y, z) \in \mathbb{R}^{2} \mid y^{3}-z^{2}=0\right\}$, if $p \in \operatorname{Int} M$ is a cusp point.

(4) Isolated point diffeomorphic to $\left\{(y, z) \in \mathbb{R}^{2} \mid y^{2}+z=0, z \geq 0\right\}$, if $p \in \partial M$ is a boundary definite fold point.

(5) Arc diffeomorphic to $\left\{(y, z) \in \mathbb{R}^{2} \mid y^{2}-z=0, z \geq 0\right\}$, if $p \in \partial M$ is a boundary indefinite fold point.

(6) Arc diffeomorphic to $\left\{(y, z) \in \mathbb{R}^{2} \mid y^{3}+z=0, z \geq 0\right\}$, if $p \in \partial M$ is a boundary cusp point.

(7) Isolated point diffeomorphic to $\left\{(y, z) \in \mathbb{R}^{2} \mid y^{2}+z^{2}=0, z \geq 0\right\}$, if $p \in$ $\partial M \cap S(f)$ is a definite $\Sigma_{1,0}^{2,0}$ point.

(8) Polygonal line diffeomorphic to $\left\{(y, z) \in \mathbb{R}^{2} \mid y^{2}-z^{2}=0, z \geq 0\right\}$, if $p \in$ $\partial M \cap S(f)$ is an indefinite $\Sigma_{1,0}^{2,0}$ point.

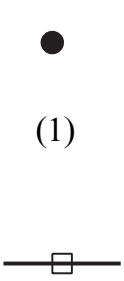

(5)

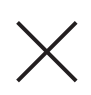

(2)

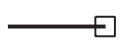

(6)

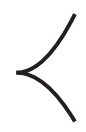

(3)

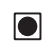

(7)
(4)

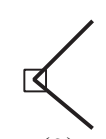

(8)

Figure 2: Neighborhoods of singular points in their singular level sets 


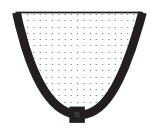

(4)

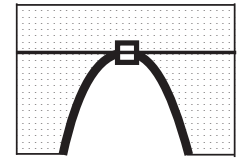

(5)

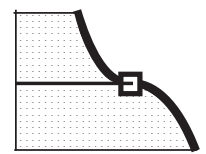

(6)

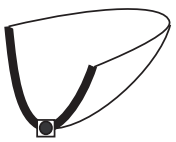

(7)

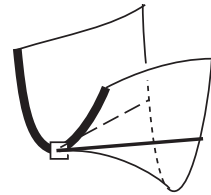

(8)

Figure 3: Singular level sets touching the boundary

Remark 2.3 In Figure 2, the black dot (1), the black square (4), and the black dot surrounded by a square (7) all represent an isolated point; however, we use distinct symbols in order to distinguish their corresponding map germs. In the figures, the squares represent points on the boundary; more precisely, they are points in $S\left(\left.f\right|_{\partial M}\right)$.

In Figure 3, singular level sets that intersect the boundary are depicted, where the surfaces appearing in the figures correspond to the hypersurface $x=0$, the intersection of the hypersurface with the boundary is depicted by thick curves, and the associated map germs restricted to the hypersurface correspond to the respective height functions. In fact, for the cases (4) and (5), the relevant map germs of 3-manifolds into surfaces are right-left equivalent to the suspensions of the function germs in the sense of Definition 4.2. As to the cases (6), (7) and (8), the relevant map germs are obtained by using certain "generic deformations" of the function germs.

For the local nearby level sets, we have the following, which can be proved by direct calculations using the corresponding normal forms.

Lemma 2.4 Let $M$ be a 3-manifold, possibly with boundary, $N$ a surface without boundary, $f: M \rightarrow N$ a proper $C^{\infty}$ stable map, and $p \in S(f) \cup S\left(\left.f\right|_{\partial M}\right)$ a singular point such that $f^{-1}(f(p)) \cap\left(S(f) \cup S\left(\left.f\right|_{\partial M}\right)\right)=\{p\}$. Then the level sets near $p$ are as depicted in Figure 4:

(1) $p \in \operatorname{Int} M$ is a definite fold point.

(2) $p \in \operatorname{Int} M$ is an indefinite fold point.

(3) $p \in \operatorname{Int} M$ is a cusp point.

(4) $p \in \partial M$ is a boundary definite fold point.

(5) $p \in \partial M$ is a boundary indefinite fold point. 
(6) $p \in \partial M$ is a boundary cusp point.

(7) $p \in S(f) \cap \partial M$ is a definite $\Sigma_{1,0}^{2,0}$ point.

(8) $p \in S(f) \cap \partial M$ is an indefinite $\Sigma_{1,0}^{2,0}$ point.

Here each of the 0-dimensional objects and the thin 1-dimensional objects represents a portion of the level set over the corresponding point in the target, each of the thick curves represents $f(S(f))$, and each of the dotted curves represents $f\left(S\left(\left.f\right|_{\partial M}\right)\right)$ near $f(p)$. Furthermore, the dotted squares represent (transverse) intersections with $\partial M$.

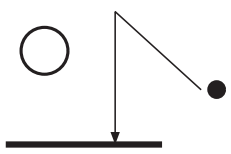

$\varnothing$

(1)

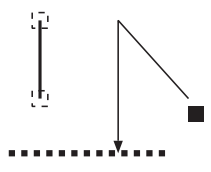

$\varnothing$

(4)

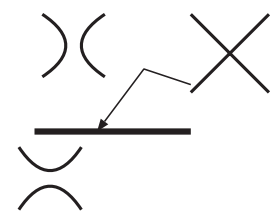

(2)

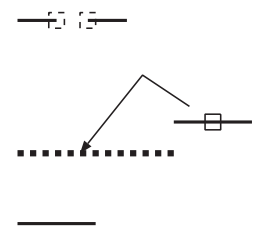

(5)

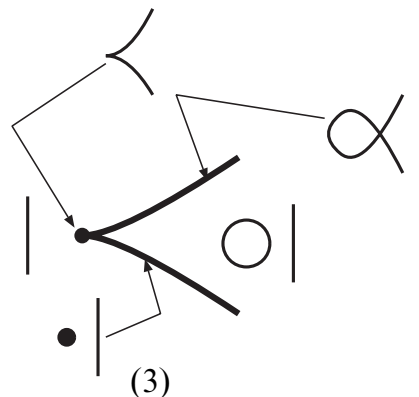

(3)

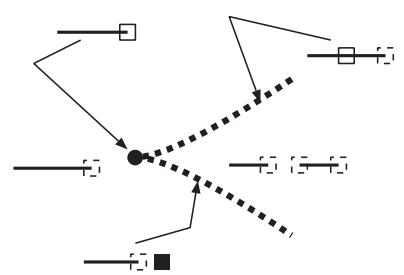

(6)

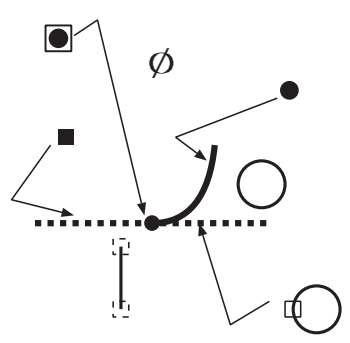

(7)

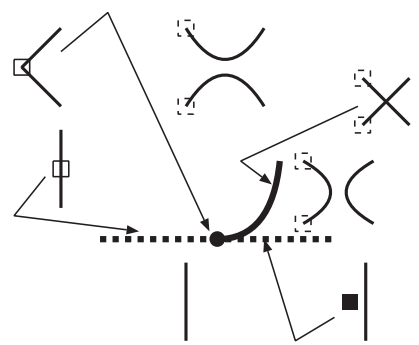

(8)

Figure 4: Local degenerations of level sets

Definition 2.5 Suppose that we are given a finite number of fibers of smooth maps, where all the dimensions of the sources and the targets are the same. Then their disjoint 
union is the fiber corresponding to the single map defined on the disjoint union of the sources, where the target spaces are all identified to a single small open disk. This definition clearly depends on such identifications; however, in the following, we can take "generic identifications" in such a way that the resulting fiber is $C^{\infty}$ stable, and the result is unique up to $C^{\infty}$ equivalence as long as these identifications are generic.

Now, by using the method developed in [7], we get the following list of singular fibers. We omit the proof here.

Proposition 2.6 (i) Each diagram in Figures 5 and 6 uniquely determines the $C^{\infty}$ equivalence class of fibers in such a way that the diagram represents the corresponding central level set, under the convention described in Remark 2.3.

(ii) Let $f: M \rightarrow N$ be a proper $C^{\infty}$ stable map of a 3-manifold $M$ with boundary into a surface $N$ without boundary. Then every fiber of $f$ is $C^{\infty}$ equivalent to the disjoint union of one of the fibers in the following list, a finite number of copies of a fiber of the trivial circle bundle, and a finite number of copies of a fiber of the trivial $I-$ bundle, where $I=[-1,1]$ :

(1) Fibers as depicted in Figure 5, ie $\widetilde{\mathrm{b} 0}^{0}, \widetilde{\mathrm{b} 0}^{1}$ and $\widetilde{\mathrm{bI}}^{\mu}$ with $2 \leq \mu \leq 10$.

(2) Fibers $\widetilde{\text { bII }}^{\mu, v}$ with $2 \leq \mu \leq v \leq 10$, where $\widetilde{\text { bII }}^{\mu, v}$ means the disjoint union of $\widetilde{\mathrm{bI}}^{\mu}$ and $\widetilde{\mathrm{bI}}^{v}$.

(3) Fibers as depicted in Figure 6, ie $\widetilde{\mathrm{bII}}^{\mu}$ with $11 \leq \mu \leq 39 \widetilde{\mathrm{bII}}^{a}, \widetilde{\mathrm{bII}}^{b}$, $\widetilde{\mathrm{bII}}^{c}$, $\widetilde{\mathrm{bII}}^{d}, \widetilde{\mathrm{bII}}^{e}$ and $\widetilde{\mathrm{bII}} f$.

More precisely, two such fibers containing no singular points of the restriction to the boundary are $C^{\infty}$ equivalent if and only if their corresponding level sets are diffeomorphic. Therefore, in the figures, the associated level sets are depicted together with the information on the corresponding local map germs, which are depicted in accordance with Lemmas 2.2 and 2.4.

In Figures 5 and $6, \kappa$ denotes the codimension of the set of points in the target $N$ whose corresponding fibers are $C^{\infty}$ equivalent to the relevant one (see [7] for details). Furthermore, the symbols $\widetilde{\mathrm{b} 0}{ }^{*}, \widetilde{\mathrm{bI}}^{*}$, and $\widetilde{\mathrm{bII}}^{*}$ mean the names of the corresponding fibers. Note that we have named the fibers so that each fiber with connected central level set has its own number or letter, and a fiber with disconnected central level set has the name consisting of the numbers of its "connected components". 


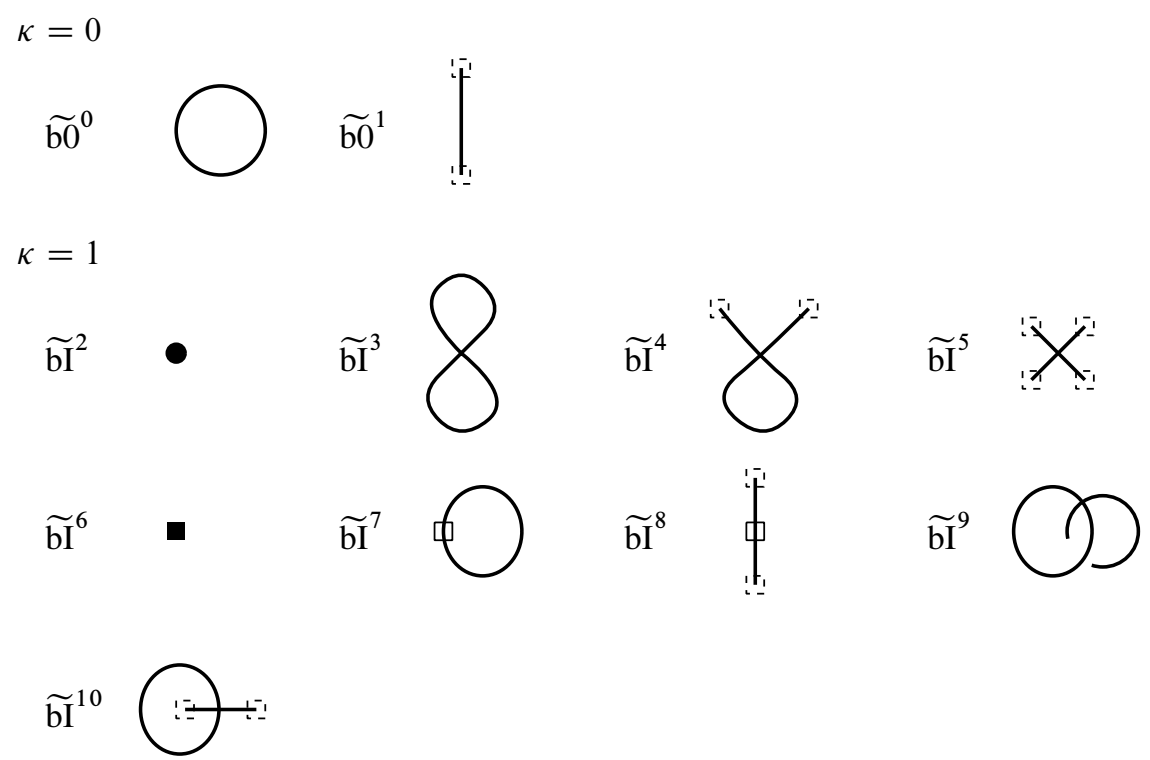

Figure 5: List of the fibers of proper $C^{\infty}$ stable maps of 3-manifolds with boundary into surfaces without boundary, for $\kappa=0,1$

Note that the fibers whose central level sets do not intersect $S\left(\left.f\right|_{\partial M}\right)$ have been essentially obtained in $[8$, Section 6].

We can prove Proposition 2.6 by using the relative version of Ehresmann's fibration theorem together with Proposition 2.1. See [7, proof of Theorem 3.5] for details.

Then we immediately obtain the following corollary. (For details, see [7, proof of Corollary 3.9].)

Corollary 2.7 Two fibers of proper $C^{\infty}$ stable maps of 3-manifolds with boundary into surfaces without boundary are $C^{\infty}$ equivalent if and only if they are $C^{0}$ equivalent.

Remark 2.8 If the source 3-manifold is orientable, then the singular fibers of types $\widetilde{\mathrm{bI}}^{9}, \widetilde{\mathrm{bI}}^{10}$ and $\widetilde{\mathrm{bII}}^{j}$ for $26 \leq j \leq 39$ never appear.

Remark 2.9 Our classification result for singular fibers of stable maps of compact orientable 3-dimensional manifolds with boundary into surfaces has already been applied in computer science. More precisely, it helps to visualize characteristic features of certain multivariate data. For details, see $[9 ; 10]$. 
$\kappa=2$

$\widetilde{\mathrm{bII}}^{11}$

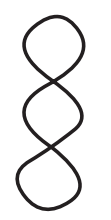

$\widetilde{\mathrm{bIII}}^{12}$

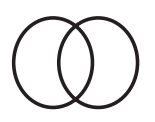

$\widetilde{\text { bII }}^{13}$
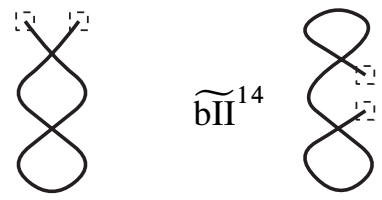

$\widetilde{\mathrm{bII}}^{15}$

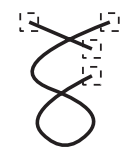

$\widetilde{\mathrm{bII}}^{16}$

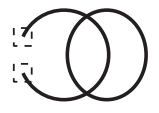

$\widetilde{\text { bII }}^{17}$<smiles>CC(C)C(C)C(C)C</smiles><smiles>[As]C1C=CC2CCC1CC2</smiles>

$\widetilde{\mathrm{bIII}}^{19}$

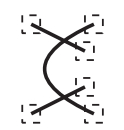

$\widetilde{\mathrm{bIII}}^{20}$<smiles>[C]1CCCCC1</smiles>

$\widetilde{\mathrm{bII}}^{21}$ 尔

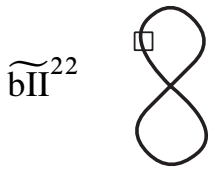

$\widetilde{\mathrm{bIII}}^{23}$<smiles>FC1(F)CCCC1</smiles>

$\widetilde{\mathrm{bIII}}^{24}$<smiles>CC1(F)CCCC1</smiles>

$\widetilde{\mathrm{bII}}^{25}$<smiles>[3H]C([3H])([3H])[3H]</smiles>

$\widetilde{\mathrm{bII}}^{26}$

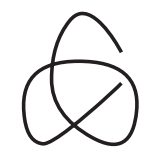

$\widetilde{\mathrm{bII}}^{27}$

$\widetilde{\mathrm{bII}}^{28}$<smiles></smiles>

$\widetilde{\text { bII }}^{29}$<smiles>C1CC2CCCC(C1)C2</smiles>

$\widetilde{\mathrm{bII}}^{30}$<smiles>FC1(F)[CH]C=CC1</smiles>

bII $^{31}$<smiles>CC1(C)CCCCC1</smiles><smiles>C1CCC2(C1)CCC2</smiles>

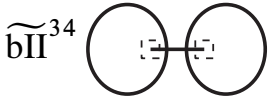

$\widetilde{\mathrm{bII}}^{35} \bigodot^{\prime \prime}$

$\widetilde{\mathrm{bII}}^{36}:=$

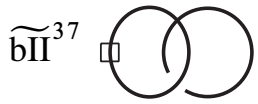

$\widetilde{\mathrm{bII}}^{38}$

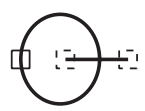

$\widetilde{\mathrm{bII}}^{39}$

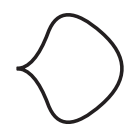

$\widetilde{\mathrm{bII}}^{b}$

$\widetilde{\mathrm{bII}}^{c}$

$\widetilde{\mathrm{bII}}^{d}$

$\widetilde{\mathrm{bII}}^{e}$

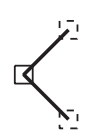

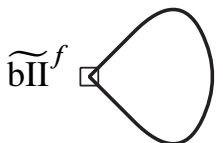

Figure 6: List of the fibers of proper $C^{\infty}$ stable maps of 3-manifolds with boundary into surfaces without boundary, for $\kappa=2$ 
Remark 2.10 Let $V$ be a surface with boundary and $W$ be the real line $\mathbb{R}$ or the circle $S^{1}$. A proper $C^{\infty}$ function $f: V \rightarrow W$ is $C^{\infty}$ stable (ie it is a stable Morse function) if and only if it satisfies the following conditions:

(1) Local conditions In the following, for $p \in \partial V$, we use local coordinates $(x, y)$ around $p$ such that Int $V$ and $\partial V$ correspond to the sets $\{y>0\}$ and $\{y=0\}$, respectively.

(1a) For $p \in \operatorname{Int} V$, the germ of $f$ at $p$ is right-left equivalent to one of the following:

$$
(x, y) \mapsto \begin{cases}x, & p: \text { regular point, } \\ x^{2} \pm y^{2}, & p: \text { fold point or nondegenerate critical point. }\end{cases}
$$

(1b) For $p \in \partial V$, the germ of $f$ at $p$ is right-left equivalent to one of the following:

$$
(x, y) \mapsto \begin{cases}x, & p: \text { regular point of }\left.f\right|_{\partial V}, \\
x^{2} \pm y, & p: \begin{array}{r}
\text { boundary fold point or } \\
\text { nondegenerate critical point of }\left.f\right|_{\partial V} .
\end{array}\end{cases}
$$

(2) Global conditions $f\left(p_{1}\right) \neq f\left(p_{2}\right)$ if $p_{1} \neq p_{2} \in S(f) \cup S\left(\left.f\right|_{\partial V}\right)$.

The list of the $C^{\infty}$ equivalence classes of singular fibers of proper stable Morse functions on surfaces with boundary can be obtained in a similar fashion. The result corresponds to those appearing in Figure 5 with $\kappa=0,1$. In fact, it is not difficult to show that the suspensions of the fibers of such functions in the sense of Definition 4.2 coincide with those appearing in the figure. However, in the following, by abuse of notation, we use the symbols in Figure 5 with $\kappa=0,1$ for the fibers of stable Morse functions as well.

\section{Coexistence of singular fibers}

Let $M$ be a compact 3-dimensional manifold with boundary, $N$ a surface without boundary, $f: M \rightarrow N$ a $C^{\infty}$ stable map, and $\tilde{\mathcal{F}}$ a $C^{\infty}$ equivalence class of singular fibers of codimension $\geq 1$. Define $\tilde{\mathcal{F}}(f)$ to be the set of points $q \in N$ such that the fiber over $q$ is $C^{\infty}$ equivalent to the disjoint union of $\tilde{\mathcal{F}}$ and some copies of a fiber of a trivial circle bundle and some copies of a fiber of a trivial $I$-bundle. Furthermore, define $\widetilde{\mathcal{F}}_{o}(f)$ (resp. $\left.\widetilde{\mathcal{F}}_{e}(f)\right)$ to be the subset of $\tilde{\mathcal{F}}(f)$ consisting of the points $q \in N$ such that the number of regular fibers, namely the total number of $\widetilde{\mathrm{b} 0}^{0}$ components and $\widetilde{\mathrm{b} 0}^{1}$ components in the fiber is odd (resp. even). For codimension-zero fibers, by convention, we denote by $\widetilde{\mathrm{b} 0}{ }_{o}(f)$ (resp. $\left.\widetilde{\mathrm{b} 0} e(f)\right)$ the set of points $q \in N$ over which lies a regular fiber consisting of an odd (resp. even) number of components. 
If $\tilde{\mathcal{F}}$ is of codimension 1 , then the closure of $\tilde{\mathcal{F}}_{o}(f)$ (or $\widetilde{\mathcal{F}}_{e}(f)$ ) is a finite graph embedded in $N$. Its vertices correspond to points over which lies a singular fiber of codimension 2 .

Notation For the rest of the section, we use the notation

$$
B_{\star}^{*}:=\left|\widetilde{\mathrm{bII}}_{\star}^{*}(f)\right|,
$$

where $|S|$ denotes the cardinality of a finite set $S$, and the map $f$ will be clear from context.

The handshake lemma from classical graph theory implies the following formulae:

Proposition 3.1 Let $f: M \rightarrow N$ be a $C^{\infty}$ stable map of a compact 3-manifold $M$ with boundary into a surface $N$ without boundary. Then the following numbers are always even:

(1) $B^{2,3}+B^{2,4}+B^{2,6}+B^{2,8}+B_{e}^{a}+B_{e}^{b}+B_{o}^{d}$

(2) $B^{2,3}+B^{2,4}+B^{2,6}+B^{2,8}+B_{o}^{a}+B_{o}^{b}+B_{e}^{d}$

(3) $B^{2,3}+B^{3,4}+B^{3,6}+B^{3,8}+B_{e}^{13}+B_{o}^{22}+B_{o}^{a}$

(4) $B^{2,3}+B^{3,4}+B^{3,6}+B^{3,8}+B_{o}^{13}+B_{e}^{22}+B_{e}^{a}$

(5) $B^{2,4}+B^{3,4}+B^{4,6}+B^{4,8}+B_{e}^{13}+B_{o}^{22}+B_{o}^{23}+B^{24}+B_{o}^{b}+B_{o}^{f}$

(6) $B^{2,4}+B^{3,4}+B^{4,6}+B^{4,8}+B_{o}^{13}+B_{e}^{22}+B_{e}^{23}+B^{24}+B_{e}^{b}+B_{e}^{f}$

(7) $B^{2,5}+B^{3,5}+B^{4,5}+B^{5,6}+B^{5,8}+B^{15}+B_{o}^{23}+B^{25}+B_{o}^{30}+B_{o}^{38}+B_{o}^{e}$

(8) $B^{2,5}+B^{3,5}+B^{4,5}+B^{5,6}+B^{5,8}+B^{15}+B_{e}^{23}+B^{25}+B_{e}^{30}+B_{e}^{38}+B_{e}^{e}$

(9) $B^{2,6}+B^{3,6}+B^{4,6}+B^{6,8}+B_{e}^{c}+B_{o}^{d}+B_{e}^{e}+B_{e}^{f}$

(10) $B^{2,6}+B^{3,6}+B^{4,6}+B^{6,8}+B_{o}^{c}+B_{e}^{d}+B_{o}^{e}+B_{o}^{f}$

(11) $B^{2,7}+B^{3,7}+B^{4,7}+B^{6,7}+B^{7,8}+B^{22}+B_{e}^{23}+B_{o}^{d}+B_{o}^{f}$

(12) $B^{2,7}+B^{3,7}+B^{4,7}+B^{6,7}+B^{7,8}+B^{22}+B_{o}^{23}+B_{e}^{d}+B_{e}^{f}$

(13) $B^{2,8}+B^{3,8}+B^{4,8}+B^{6,8}+B_{o}^{23}+B^{24}+B_{o}^{c}+B_{o}^{e}$

(14) $B^{2,8}+B^{3,8}+B^{4,8}+B^{6,8}+B_{e}^{23}+B^{24}+B_{e}^{c}+B_{e}^{e}$

(15) $B^{2,9}+B^{3,9}+B^{4,9}+B^{6,9}+B^{8,9}+B^{27}+B_{e}^{35}+B_{o}^{37}$

(16) $B^{2,9}+B^{3,9}+B^{4,9}+B^{6,9}+B^{8,9}+B^{27}+B_{o}^{35}+B_{e}^{37}$

(17) $B^{2,10}+B^{3,10}+B^{4,10}+B^{6,10}+B^{8,10}+B_{e}^{30}+B^{32}+B^{33}+B_{o}^{35}+B_{o}^{37}$

$+B_{o}^{38}+B^{39}$

(18) $B^{2,10}+B^{3,10}+B^{4,10}+B^{6,10}+B^{8,10}+B_{0}^{30}+B^{32}+B^{33}+B_{e}^{35}+B_{e}^{37}$ $+B_{e}^{38}+B^{39}$ 
By eliminating the terms of the forms $\mathcal{F}_{o}(f)$ and $\mathcal{F}_{e}(f)$, we obtain the following:

Corollary 3.2 Let $f: M \rightarrow N$ be a $C^{\infty}$ stable map of a compact 3-manifold $M$ with boundary into a surface $N$ without boundary. Then the following numbers are always even:
(1) $B^{a}+B^{b}+B^{d}$
(2) $B^{13}+B^{22}+B^{a}$
(3) $B^{13}+B^{22}+B^{23}+B^{b}+B^{f}$
(4) $B^{23}+B^{30}+B^{38}+B^{e}$
(5) $B^{c}+B^{d}+B^{e}+B^{f}$
(6) $B^{23}+B^{d}+B^{f}$
(7) $B^{23}+B^{c}+B^{e}$
(8) $B^{35}+B^{37}$
(9) $B^{30}+B^{35}+B^{37}+B^{38}$

Remark 3.3 The numbers in Corollary 3.2 are all even if and only if the following seven are all even:
(1) $B^{a}+B^{b}+B^{d}$
(2) $B^{13}+B^{22}+B^{a}$
(3) $B^{23}+B^{e}$
(4) $B^{c}$
(5) $B^{d}+B^{e}+B^{f}$
(6) $B^{35}+B^{37}$
(7) $B^{30}+B^{38}$

Remark 3.4 By using the same method, we obtain similar coexistence results for singular fibers of a stable Morse function $f: V \rightarrow W$ of a compact surface $V$ with boundary into $W=\mathbb{R}$ or $S^{1}$ :

$$
\left|\widetilde{\mathrm{bI}}^{2}(f)\right|+\left|\widetilde{\mathrm{bI}}^{3}(f)\right|+\left|\widetilde{\mathrm{bI}}^{4}(f)\right|+\left|\widetilde{\mathrm{bI}}^{6}(f)\right|+\left|\widetilde{\mathrm{bI}}^{8}(f)\right| \equiv 0 \quad \bmod 2,
$$

where we are using the notation for the relevant fibers in the sense of Remark 2.10. 
For a stable Morse function $f: V \rightarrow W$ as above and a $C^{\infty}$ equivalence class $\tilde{\mathcal{F}}$ of singular fibers, denote by $\widetilde{\mathcal{F}}_{o, o}(f)$ (or $\widetilde{\mathcal{F}}_{o, e}(f), \widetilde{\mathcal{F}}_{e, o}(f), \widetilde{\mathcal{F}}_{e, e}(f)$ ) the subset of $\tilde{\mathcal{F}}(f)$ which consists of the points $q \in W$ such that the numbers of fibers of types $\widetilde{\mathrm{b} 0}{ }^{0}$ and $\widetilde{\mathrm{b} 0}^{1}$ are both odd (resp. odd and even, even and odd, or both even). Then, by applying the same method to the graph obtained as the closure of $\widetilde{\mathcal{F}}_{*, \star}(f)(*, \star=o$ or $e$ ), we obtain the following:

Lemma 3.5 Let $f: V \rightarrow W$ be a stable Morse function on a compact surface $V$ with boundary into $W=\mathbb{R}$ or $S^{1}$. Then the following numbers are always even:

$$
\begin{aligned}
& \left|\widetilde{\mathrm{bI}}^{2}(f)\right|+\left|\widetilde{\mathrm{bI}}^{3}(f)\right|+\left|\widetilde{\mathrm{bI}}^{4}(f)\right|+\left|\widetilde{\mathrm{bI}}^{7}(f)\right| \\
& \left|\widetilde{\mathrm{bI}}^{6}(f)\right|+\left|\widetilde{\mathrm{bI}}^{7}(f)\right|+\left|\widetilde{\mathrm{bI}}^{8}(f)\right|
\end{aligned}
$$

\section{Universal complex}

In this section we review the theory of the universal complex of singular fibers and then study the universal complex for stable maps of manifolds with boundary. For the general theory of universal complex of singular fibers, refer to [7, Chapters 7-8].

Throughout this section, for a $C^{\infty}$ map $M \rightarrow N$, we assume that $M$ is an $m-$ dimensional manifold which is not necessarily closed, and that $N$ is an $n$-dimensional manifold without boundary.

In what follows, the codimension of a smooth map $f: M \rightarrow N$ refers to the difference $\operatorname{dim} N-\operatorname{dim} M \in \mathbb{Z}$. To construct the universal complex of singular fibers of $C^{\infty}$ maps, we fix an integer $\ell \in \mathbb{Z}$ for the codimension of the maps, and consider

(1) a set $\tau$ of fibers of proper Thom maps ${ }^{1}$ of codimension $\ell$, and

(2) an equivalence relation $\rho$ among the fibers in $\tau$.

We further assume that the set $\tau$ and the relation $\rho$ satisfy the following conditions:

(a) The set $\tau$ is closed under adjacency relation. That is, if a fiber is in $\tau$, then so are all nearby fibers.

(b) The relation $\rho$ is weaker than the $C^{0}$ equivalence: each $\rho$-class is a union of $C^{0}$ equivalence classes.

${ }^{1}$ A Thom map $M \rightarrow N$ is a $C^{\infty}$ stratified map with respect to Whitney regular stratifications of $M$ and $N$ which is a submersion on each stratum and satisfies certain regularity conditions. See [1, Section 2; 6, Section 2.5; 2; 7, Part I, Section 1], etc for more details. 
(c) Let $f_{i}: M_{i} \rightarrow N_{i}$ be proper Thom maps and $q_{i} \in N_{i}, i=0,1$. Suppose that the fibers over $q_{0}$ and $q_{1}$ are in $\tau$ and that they are equivalent with respect to $\rho$. Then there exist open neighborhoods $U_{i}$ of $q_{i}$ in $N_{i}, i=0,1$, and a homeomorphism $\varphi: U_{0} \rightarrow U_{1}$ satisfying $\varphi\left(q_{0}\right)=q_{1}$ and $\varphi\left(U_{0} \cap \mathcal{F}\left(f_{0}\right)\right)=U_{1} \cap \mathcal{F}\left(f_{1}\right)$, for each $\rho$-class $\mathcal{F}$, where for a proper Thom map $f: M \rightarrow N$ of codimension $\ell$, we set

$$
\mathcal{F}(f)=\{q \in N \mid \text { the fiber of } f \text { over } q \text { belongs to the class } \mathcal{F}\} .
$$

In particular, these conditions imply that, for each proper Thom map $f: M \rightarrow N$ and each $\rho$-class $\mathcal{F}, \mathcal{F}(f)$ is a $C^{0}$ submanifold of constant codimension unless it is empty. The codimension of $\mathcal{F}$ is defined to be that of $\mathcal{F}(f)$ in $N$, and is denoted by $\kappa(\mathcal{F})$.

We call a proper Thom map $f: M \rightarrow N$ a $\tau$-map if all of its fibers are in $\tau$.

For each $\kappa \in \mathbb{Z}$, let $C^{\kappa}(\tau, \rho)$ be the formal $\mathbb{Z}_{2}$-vector space spanned by the $\rho$-classes of codimension $\kappa$ in $\tau$. If there are no such fibers, then set $C^{\kappa}(\tau, \rho)=0$. Note that $C^{\kappa}(\tau, \rho)$ may possibly be of infinite dimension in general.

Define the $\mathbb{Z}_{2}$-linear map $\delta_{\kappa}: C^{\kappa}(\tau, \rho) \rightarrow C^{\kappa+1}(\tau, \rho)$ by

$$
\delta_{\kappa}(\mathcal{F})=\sum_{\kappa(\mathcal{G})=\kappa+1} n_{\mathcal{F}}(\mathcal{G}) \mathcal{G}
$$

where $n_{\mathcal{F}}(\mathcal{G}) \in \mathbb{Z}_{2}$ is the number modulo two of the local components of $\mathcal{F}(f)$ which are locally adjacent to a component of $\mathcal{G}(f)$ for a $\tau$-map $f$ satisfying $\mathcal{G}(f) \neq \varnothing$ (for details, see [7]). Note that the coefficient $n_{\mathcal{F}}(\mathcal{G}) \in \mathbb{Z}_{2}$ is well-defined by condition (c) for $\rho$.

Since we see easily that $\delta_{\kappa+1} \circ \delta_{\kappa}=0$ holds, we obtain the cochain complex

$$
\mathcal{C}(\tau, \rho)=\left(C^{\kappa}(\tau, \rho), \delta_{\kappa}\right)_{\kappa} .
$$

We call the resulting cochain complex the universal complex of singular fibers for proper $\tau$-maps with respect to the equivalence relation $\rho$, and denote its cohomology group of dimension $\kappa$ by $H^{\kappa}(\tau, \rho)$.

Let

$$
c=\sum_{\kappa(\mathcal{F})=\kappa} n_{\mathcal{F}} \mathcal{F}, \quad n_{\mathcal{F}} \in \mathbb{Z}_{2}
$$

be a $\kappa$-dimensional cochain of $\mathcal{C}(\tau, \rho)$. For a $\tau$-map $f: M \rightarrow N$, denote by $c(f)$ the closure of the set of points $q \in N$ such that the fiber over $q$ is in $\mathcal{F}$ with $n_{\mathcal{F}} \neq 0$. If $c$ is a cocycle, then we can show that $c(f)$ is a $\mathbb{Z}_{2}$-cycle of closed support of codimension $\kappa$ in $N$. In addition, if $M$ is compact and $\kappa>0$, then $c(f)$ is a $\mathbb{Z}_{2}$-cycle in the usual sense. 
Lemma 4.1 Let $c$ and $c^{\prime}$ be $\kappa$-dimensional cocycles of $\mathcal{C}(\tau, \rho)$. If they are cohomologous, then the $\mathbb{Z}_{2}$-cycles $c(f)$ and $c^{\prime}(f)$ are $\mathbb{Z}_{2}$-homologous in $N$ for each $\tau$-map $f: M \rightarrow N$.

Proof There exists a $(\kappa-1)$-dimensional cochain $d$ of $\mathcal{C}(\tau, \rho)$ such that $c-c^{\prime}=$ $\delta_{\kappa-1} d$. Then we see easily that $c(f)-c^{\prime}(f)=\partial d(f)$ holds, and the result follows immediately.

Let $[c]$ be the $\kappa$-dimensional cohomology class of $\mathcal{C}(\tau, \rho)$ represented by a cochain $c$. For a $\tau$-map $f: M \rightarrow N$, define $[c(f)] \in H_{n-\kappa}^{c}\left(N ; \mathbb{Z}_{2}\right)$ to be the $\mathbb{Z}_{2}$-homology class represented by the $\mathbb{Z}_{2}$-cycle $c(f)$ of closed support, where $H_{*}^{c}$ denotes the Borel-Moore homology or the homology with closed support. This is well-defined by virtue of Lemma 4.1.

Define the $\mathbb{Z}_{2}$-linear map

$$
\varphi_{f}: H^{\kappa}(\tau, \rho) \rightarrow H^{\kappa}\left(N ; \mathbb{Z}_{2}\right), \quad \varphi_{f}([c])=[c(f)]^{*},
$$

where $[c(f)]^{*} \in H^{\kappa}\left(N ; \mathbb{Z}_{2}\right)$ is the Poincaré dual of $[c(f)] \in H_{n-\kappa}^{c}\left(N ; \mathbb{Z}_{2}\right)$.

The suspension of a Thom map is introduced as follows:

Definition 4.2 For a proper Thom map $f: M \rightarrow N$, consider the proper Thom map

$$
f \times \operatorname{id}_{\mathbb{R}}: M \times \mathbb{R} \rightarrow N \times \mathbb{R} .
$$

Note that $S\left(f \times \operatorname{id}_{\mathbb{R}}\right)=S(f) \times \mathbb{R}$ and $\left(f \times \operatorname{id}_{\mathbb{R}}\right)(S(f) \times \mathbb{R})=f(S(f)) \times \mathbb{R}$. We call $f \times \operatorname{id}_{\mathbb{R}}$ and the fiber of $f \times \operatorname{id}_{\mathbb{R}}$ over a point $(q, 0) \in N \times \mathbb{R}$ the suspension of $f$ and the suspension of the fiber of $f$ over $q \in N$, respectively.

Let $\tau$ be a set of fibers for proper Thom maps of codimension $\ell$. For a dimension pair ( $m, n)$ with $n-m=\ell$, let $\tau(m, n)$ denote the set of fibers in $\tau$ for proper Thom maps of manifolds of dimension $m$ into those of dimension $n$. The equivalence relation on $\tau(m, n)$ induced by $\rho$ is denoted by $\rho_{m, n}$.

In the following, in addition to conditions (a)-(c) above, we assume the following two additional conditions:

(d) The suspension of each fiber in $\tau(m, n)$ is also in $\tau(m+1, n+1)$.

(e) If two fibers in $\tau(m, n)$ are equivalent with respect to $\rho_{m, n}$, then their suspensions are also equivalent with respect to $\rho_{m+1, n+1}$. 
For each $\kappa \in \mathbb{Z}$, the suspension induces the $\mathbb{Z}_{2}$-linear map

$$
s_{\kappa}: C^{\kappa}\left(\tau(m+1, n+1), \rho_{m+1, n+1}\right) \rightarrow C^{\kappa}\left(\tau(m, n), \rho_{m, n}\right),
$$

where $s_{\kappa}(\mathcal{F})$ is the (possibly infinite) sum of all $\rho_{m, n}$-classes of codimension $\kappa$ whose suspensions are in $\mathcal{F}$. Note that $s_{\kappa}$ is well-defined.

Lemma 4.3 The system of $\mathbb{Z}_{2}$-linear maps $\left\{s_{\kappa}\right\}$ defines the cochain map

$$
\left\{s_{\kappa}\right\}: \mathcal{C}\left(\tau(m+1, n+1), \rho_{m+1, n+1}\right) \rightarrow \mathcal{C}\left(\tau(m, n), \rho_{m, n}\right) .
$$

We omit the proof of this lemma. For details, see [7].

Let us now introduce a geometric equivalence relation for $\tau$-maps.

Definition 4.4 Two $\tau$-maps $f_{i}: M_{i} \rightarrow N, i=0,1$, of compact manifolds with boundary into a manifold without boundary are $\tau$-cobordant if there exist a compact manifold $X$ with corners and a smooth map $F: X \rightarrow N \times[0,1]$ that satisfy the following conditions:

(1) $\partial X=M_{0} \cup Q \cup M_{1}$, where $M_{0}, M_{1}$ and $Q$ are codimension 0 smooth submanifolds of $\partial X, M_{0} \cap M_{1}=\varnothing$, and $\partial Q=\left(M_{0} \cap Q\right) \cup\left(M_{1} \cap Q\right)$,

(2) $X$ has corners along $\partial Q$,

(3) $\left.F\right|_{M_{0} \times[0, \varepsilon)}=f_{0} \times \operatorname{id}_{[0, \varepsilon)}$ and $\left.F\right|_{M_{1} \times(1-\varepsilon, 1]}=f_{1} \times \operatorname{id}_{(1-\varepsilon, 1]}$, where $M_{0} \times[0, \varepsilon)$ and $M_{1} \times(1-\varepsilon, 1]$ denote the collar neighborhoods (with corners) of $M_{0}$ and $M_{1}$ in $X$, respectively,

$F^{-1}(N \times\{i\})=N_{i}, i=0,1$, and the restriction

$$
\left.F\right|_{X \backslash\left(M_{0} \cup M_{1}\right)}: X \backslash\left(M_{0} \cup M_{1}\right) \rightarrow N \times(0,1)
$$

is a $\tau$-map.

In this case, we call the map $F$ a $\tau$-cobordism between $f_{0}$ and $f_{1}$.

Note that the $\tau$-cobordism relation is an equivalence relation among the $\tau$-maps into a fixed manifold $N$. For a manifold $N$, we denote by $\operatorname{Cob}_{\tau}(N)$ the set of all equivalence classes of $\tau$-maps of compact manifolds into $N$ with respect to the $\tau$-cobordism.

Proposition 4.5 If two $\tau$-maps $f_{i}: M_{i} \rightarrow N$ of compact manifolds into $N, i=0,1$, are $\tau$-cobordant, then for each $\kappa$ we have

$$
\left.\varphi_{f_{0}}\right|_{\operatorname{Im} s_{\kappa *}}=\left.\varphi_{f_{1}}\right|_{\operatorname{Im} s_{\kappa *}}: \operatorname{Im} s_{\kappa *} \rightarrow H^{\kappa}\left(N ; \mathbb{Z}_{2}\right),
$$

where $s_{\kappa *}: H^{\kappa}\left(\tau(m+1, n+1), \rho_{m+1, n+1}\right) \rightarrow H^{\kappa}\left(\tau(m, n), \rho_{m, n}\right)$ denotes the homomorphism induced by the suspension. 
Proof Let $F: X \rightarrow N \times[0,1]$ be a $\tau$-cobordism between $f_{0}$ and $f_{1}$. For each $\kappa$-dimensional cocycle $c=\sum_{\kappa(\mathcal{F})=\kappa} n_{\mathcal{F}} \mathcal{F}$ of $\mathcal{C}\left(\tau(m+1, n+1), \rho_{m+1, n+1}\right)$, put $\bar{c}=s_{\kappa}(c) \in C^{\kappa}\left(\tau(m, n), \rho_{m, n}\right)$. Then we have

$$
\partial c(F)=\bar{c}\left(f_{1}\right) \times\{1\}-\bar{c}\left(f_{0}\right) \times\{0\},
$$

since $c$ is a cocycle, where $c(F)$ denotes the closure in $N \times[0,1]$ of the set of points $(q, t) \in N \times(0,1)$ such that the fiber over $(q, t)$ is in $\mathcal{F}$ with $n_{\mathcal{F}} \neq 0$. Then the result follows immediately.

Thus, for each cohomology class $[c] \in H^{\kappa}\left(\tau(m+1, n+1), \rho_{m+1, n+1}\right)$ and an $n-$ dimensional manifold $N$ without boundary, we obtain the map

$$
I_{[c]}: \operatorname{Cob}_{\tau}(N) \rightarrow H^{\kappa}\left(N ; \mathbb{Z}_{2}\right)
$$

defined by $I_{[c]}(f)=\varphi_{f}\left(s_{\kappa *}[c]\right)$, which does not depend on the choice of a representative $f$ of a given $\tau$-cobordism class. Namely, each element in

$$
H^{\kappa}\left(\tau(m+1, n+1), \rho_{m+1, n+1}\right)
$$

induces a $\tau$-cobordism invariant for $\tau$-maps into an $n$-dimensional manifold $N$.

\section{Universal complex for stable maps of $\boldsymbol{n}$-dimensional manifolds with boundary into $(n-1)$-dimensional manifolds}

For a positive integer $n$, let $b \mathcal{S}_{\mathrm{pr}}(n, n-1)$ be the set of fibers for proper $C^{0}$ stable Thom maps of $n$-dimensional manifolds with boundary into $(n-1)$-dimensional manifolds without boundary. We put

$$
b \mathcal{S}_{\mathrm{pr}}=\bigcup_{n=1}^{\infty} b \mathcal{S}_{\mathrm{pr}}(n, n-1)
$$

Remark 4.6 If the dimension pair $(n, n-1)$ is in the nice range, then $C^{0}$ stable maps are $C^{\infty}$ stable (for example, see [6]), and consequently they are Thom maps. For example, this is the case if $n \leq 8$.

Furthermore, let $\rho_{n, n-1}(2)$ be the $C^{0}$ equivalence relation modulo two regular fibers for fibers in $b \mathcal{S}_{\mathrm{pr}}(n, n-1)$, ie two fibers in $b \mathcal{S}_{\mathrm{pr}}(n, n-1)$ are $\rho_{n, n-1}(2)$ equivalent if they become $C^{0}$ equivalent after we add some regular fibers to each of them with the numbers of added components having the same parity. Note that, under this equivalence, for $n=2,3$, we do not distinguish the fibers of types $\widetilde{\mathrm{b} 0}^{0}$ from those of type $\widetilde{\mathrm{b} 0}{ }^{1}$. Therefore, in the following, we denote both of them by $\widetilde{\mathrm{b} 0}$. 
We denote by $\rho(2)$ the equivalence relation on $b \mathcal{S}_{\text {pr }}$ induced by $\rho_{n, n-1}(2), n \geq 1$. Note that the set $b \mathcal{S}_{\text {pr }}$ and the equivalence relation $\rho(2)$ satisfy conditions (a)-(e) described above.

For a $C^{0}$ equivalence class $\tilde{\mathcal{F}}$ of singular fibers, denote by $\widetilde{\mathcal{F}}_{o}$ (resp. $\widetilde{\mathcal{F}}_{e}$ ) the equivalence class with respect to $\rho_{n, n-1}(2)$ consisting of singular fibers of type $\tilde{\mathcal{F}}$ with an odd (resp. even) number of regular fiber components. For $n=2,3$, we denote by $\widetilde{\mathrm{b}}_{o}$ (resp. $\widetilde{\mathrm{b} 0}{ }_{e}$ ) the equivalence class with respect to $\rho_{n, n-1}(2)$ consisting exclusively of an odd (resp. even) number of regular fiber components.

Then, for the universal complex $\mathcal{C}\left(b \mathcal{S}_{\text {pr }}(3,2), \rho_{3,2}(2)\right)$, the coboundary homomorphism is given by the following formulae, which are obtained with the help of Lemma 2.4:

$$
\begin{aligned}
& \delta_{0}\left(\widetilde{\mathrm{b}}_{o}\right)=\delta_{0}\left(\widetilde{\mathrm{b}}_{e}\right)=\widetilde{\mathrm{bI}}^{2}+\widetilde{\mathrm{bI}}^{3}+\widetilde{\mathrm{bI}}^{4}+\widetilde{\mathrm{bI}}^{6}+\widetilde{\mathrm{bI}}^{8}, \\
& \delta_{1}\left(\widetilde{\mathrm{bI}}_{o}^{2}\right)=\widetilde{\mathrm{bII}}^{2,3}+\widetilde{\mathrm{bII}}^{2,4}+\widetilde{\mathrm{bII}}^{2,6}+\widetilde{\mathrm{bII}}^{2,8}+\widetilde{\mathrm{bII}}_{e}^{a}+\widetilde{\mathrm{bII}}_{e}^{b}+\widetilde{\mathrm{bII}}_{o}^{d}, \\
& \delta_{1}\left(\widetilde{\mathrm{bI}}_{e}^{2}\right)=\widetilde{\mathrm{bII}}^{2,3}+\widetilde{\mathrm{bII}}^{2,4}+\widetilde{\mathrm{bII}}^{2,6}+\widetilde{\mathrm{bII}}^{2,8}+\widetilde{\mathrm{bII}}_{o}^{a}+\widetilde{\mathrm{bII}}_{o}^{b}+\widetilde{\mathrm{bII}}_{e}^{d}, \\
& \delta_{1}\left(\widetilde{\mathrm{bl}}_{o}^{3}\right)=\widetilde{\mathrm{bII}}^{2,3}+\widetilde{\mathrm{bII}}^{3,4}+\widetilde{\mathrm{bII}}^{3,6}+\widetilde{\mathrm{bII}}^{3,8}+\widetilde{\mathrm{bII}}_{e}^{13}+\widetilde{\mathrm{bII}}_{o}^{22}+\widetilde{\mathrm{bII}}_{o}^{a}, \\
& \delta_{1}\left(\widetilde{\mathrm{bl}}_{e}^{3}\right)=\widetilde{\mathrm{bII}}^{2,3}+\widetilde{\mathrm{bII}}^{3,4}+\widetilde{\mathrm{bII}}^{3,6}+\widetilde{\mathrm{bII}}^{3,8}+\widetilde{\mathrm{bII}}_{o}^{13}+\widetilde{\mathrm{bII}}_{e}^{22}+\widetilde{\mathrm{bII}}_{e}^{a}, \\
& \delta_{1}\left(\widetilde{\mathrm{bI}}_{o}^{4}\right)=\widetilde{\mathrm{bII}}^{2,4}+\widetilde{\mathrm{bII}}^{3,4}+\widetilde{\mathrm{bII}}^{4,6}+\widetilde{\mathrm{bII}}^{4,8}+\widetilde{\mathrm{bII}}_{e}^{13}+\widetilde{\mathrm{bII}}_{o}^{22}+\widetilde{\mathrm{bII}}_{o}^{23}+\widetilde{\mathrm{bII}}^{24} \\
& +\widetilde{\mathrm{bII}}_{o}^{b}+\widetilde{\mathrm{bII}}_{o}^{f} \text {, } \\
& \delta_{1}\left(\widetilde{\mathrm{bI}}_{e}^{4}\right)=\widetilde{\mathrm{bII}}^{2,4}+\widetilde{\mathrm{bII}}^{3,4}+\widetilde{\mathrm{bII}}^{4,6}+\widetilde{\mathrm{bII}}^{4,8}+\widetilde{\mathrm{bII}}_{o}^{13}+\widetilde{\mathrm{bII}}_{e}^{22}+\widetilde{\mathrm{bII}}_{e}^{23}+\widetilde{\mathrm{bII}}^{24} \\
& +\widetilde{\mathrm{bII}}_{e}^{b}+\widetilde{\mathrm{bII}}_{e}^{f} \\
& \delta_{1}\left(\widetilde{\mathrm{bl}}_{o}^{5}\right)=\widetilde{\mathrm{bII}}^{2,5}+\widetilde{\mathrm{bII}}^{3,5}+\widetilde{\mathrm{bII}}^{4,5}+\widetilde{\mathrm{bII}}^{5,6}+\widetilde{\mathrm{bII}}^{5,8}+\widetilde{\mathrm{bII}}^{15}+\widetilde{\mathrm{bII}}_{o}^{23}+\widetilde{\mathrm{bII}}^{25} \\
& +\widetilde{\mathrm{bII}}_{o}^{30}+\widetilde{\mathrm{bII}}_{o}^{38}+\widetilde{\mathrm{bII}}_{o}^{e}, \\
& \delta_{1}\left(\widetilde{\mathrm{bI}}_{e}^{5}\right)=\widetilde{\mathrm{bII}}^{2,5}+\widetilde{\mathrm{bII}}^{3,5}+\widetilde{\mathrm{bII}}^{4,5}+\widetilde{\mathrm{bII}}^{5,6}+\widetilde{\mathrm{bII}}^{5,8}+\widetilde{\mathrm{bII}}^{15}+\widetilde{\mathrm{bII}}_{e}^{23}+\widetilde{\mathrm{bII}}^{25} \\
& +\widetilde{\mathrm{bII}}_{e}^{30}+\widetilde{\mathrm{bII}}_{e}^{38}+\widetilde{\mathrm{bII}}_{e}^{e}, \\
& \delta_{1}\left(\widetilde{\mathrm{bl}}_{o}^{6}\right)=\widetilde{\mathrm{bII}}^{2,6}+\widetilde{\mathrm{bII}}^{3,6}+\widetilde{\mathrm{bII}}^{4,6}+\widetilde{\mathrm{bII}}^{6,8}+\widetilde{\mathrm{bII}}_{e}^{c}+\widetilde{\mathrm{bII}}_{o}^{d}+\widetilde{\mathrm{bII}}_{e}^{e}+\widetilde{\mathrm{bII}}_{e}^{f}, \\
& \delta_{1}\left(\widetilde{\mathrm{bl}}_{e}^{6}\right)=\widetilde{\mathrm{bII}}^{2,6}+\widetilde{\mathrm{bII}}^{3,6}+\widetilde{\mathrm{bII}}^{4,6}+\widetilde{\mathrm{bII}}^{6,8}+\widetilde{\mathrm{bII}}_{o}^{c}+\widetilde{\mathrm{bII}}_{e}^{d}+\widetilde{\mathrm{bII}}_{o}^{e}+\widetilde{\mathrm{bII}}_{o}^{f}, \\
& \delta_{1}\left(\widetilde{\mathrm{bI}}_{o}^{7}\right)=\widetilde{\mathrm{bII}}^{2,7}+\widetilde{\mathrm{bII}}^{3,7}+\widetilde{\mathrm{bII}}^{4,7}+\widetilde{\mathrm{bII}}^{6,7}+\widetilde{\mathrm{bII}}^{7,8}+\widetilde{\mathrm{bII}}^{22}+\widetilde{\mathrm{bII}}_{e}^{23}+\widetilde{\mathrm{bII}}_{o}^{d}+\widetilde{\mathrm{bII}}_{o}^{f}, \\
& \delta_{1}\left(\widetilde{\mathrm{bI}}_{e}^{7}\right)=\widetilde{\mathrm{bII}}^{2,7}+\widetilde{\mathrm{bII}}^{3,7}+\widetilde{\mathrm{bII}}^{4,7}+\widetilde{\mathrm{bII}}^{6,7}+\widetilde{\mathrm{bII}}^{7,8}+\widetilde{\mathrm{bII}}^{22}+\widetilde{\mathrm{bII}}_{o}^{23}+\widetilde{\mathrm{bII}}_{e}^{d}+\widetilde{\mathrm{bII}}_{e}^{f}, \\
& \delta_{1}\left(\widetilde{\mathrm{bI}}_{o}^{8}\right)=\widetilde{\mathrm{bII}}^{2,8}+\widetilde{\mathrm{bII}}^{3,8}+\widetilde{\mathrm{bII}}^{4,8}+\widetilde{\mathrm{bII}}^{6,8}+\widetilde{\mathrm{bII}}_{o}^{23}+\widetilde{\mathrm{bII}}^{24}+\widetilde{\mathrm{bII}}_{o}^{c}+\widetilde{\mathrm{bII}}_{o}^{e},
\end{aligned}
$$




$$
\begin{aligned}
\delta_{1}\left(\widetilde{\mathrm{bI}}_{e}^{8}\right)=\widetilde{\mathrm{bII}}^{2,8}+\widetilde{\mathrm{bII}}^{3,8}+\widetilde{\mathrm{bII}}^{4,8}+\widetilde{\mathrm{bII}}^{6,8}+\widetilde{\mathrm{bII}}_{e}^{23}+\widetilde{\mathrm{bII}}^{24}+\widetilde{\mathrm{bII}}_{e}^{c}+\widetilde{\mathrm{bII}}_{e}^{e}, \\
\delta_{1}\left(\widetilde{\mathrm{bI}}_{o}^{9}\right)=\widetilde{\mathrm{bII}}^{2,9}+\widetilde{\mathrm{bII}}^{3,9}+\widetilde{\mathrm{bII}}^{4,9}+\widetilde{\mathrm{bII}}^{6,9}+\widetilde{\mathrm{bII}}^{8,9}+\widetilde{\mathrm{bII}}^{27}+\widetilde{\mathrm{bII}}_{e}^{35}+\widetilde{\mathrm{bII}}_{o}^{37}, \\
\delta_{1}\left(\widetilde{\mathrm{bI}}_{e}^{9}\right)=\widetilde{\mathrm{bII}}^{2,9}+\widetilde{\mathrm{bII}}^{3,9}+\widetilde{\mathrm{bII}}^{4,9}+\widetilde{\mathrm{bII}}^{6,9}+\widetilde{\mathrm{bII}}^{8,9}+\widetilde{\mathrm{bII}}^{27}+\widetilde{\mathrm{bII}}_{o}^{35}+\widetilde{\mathrm{bII}}_{e}^{37}, \\
\delta_{1}\left(\widetilde{\mathrm{bI}}_{o}^{10}\right)=\widetilde{\mathrm{bII}}^{2,10}+\widetilde{\mathrm{bII}}^{3,10}+\widetilde{\mathrm{bII}}^{4,10}+\widetilde{\mathrm{bII}}^{6,10}+\widetilde{\mathrm{bII}}^{8,10}+\widetilde{\mathrm{bII}}_{e}^{30}+\widetilde{\mathrm{bII}}^{32} \\
\quad+\widetilde{\mathrm{bII}}^{33}+\widetilde{\mathrm{bII}}_{o}^{35}+\widetilde{\mathrm{bII}}_{o}^{37}+\widetilde{\mathrm{bII}}_{o}^{38}+\widetilde{\mathrm{bII}}^{39}, \\
\delta_{1}\left(\widetilde{\mathrm{bI}}_{e}^{10}\right)=\widetilde{\mathrm{bII}}^{2,10}+\widetilde{\mathrm{bII}}^{3,10}+\widetilde{\mathrm{bII}}^{4,10}+\widetilde{\mathrm{bII}}^{6,10}+\widetilde{\mathrm{bII}}^{8,10}+\widetilde{\mathrm{bII}}_{o}^{30}+\widetilde{\mathrm{bII}}^{32} \\
\quad+\widetilde{\mathrm{bII}}^{33}+\widetilde{\mathrm{bII}}_{e}^{35}+\widetilde{\mathrm{bII}}_{e}^{37}+\widetilde{\mathrm{bII}}_{e}^{38}+\widetilde{\mathrm{bII}}^{39},
\end{aligned}
$$

where $\tilde{\mathcal{F}}$ denotes $\tilde{\mathcal{F}}_{o}+\tilde{\mathcal{F}}_{e}$.

Then, by a straightforward calculation, we obtain the following:

Proposition 4.7 The cohomology groups of $\mathcal{C}\left(b \mathcal{S}_{\mathrm{pr}}(3,2), \rho_{3,2}(2)\right)$ are described as follows:

(1) $H^{0}\left(b \mathcal{S}_{\mathrm{pr}}(3,2), \rho_{3,2}(2)\right) \cong \mathbb{Z}_{2}$, generated by $\left[\widetilde{\mathrm{b} 0}_{o}+\widetilde{\mathrm{b} 0}_{e}\right]$.

(2) $H^{1}\left(b \mathcal{S}_{\text {pr }}(3,2), \rho_{3,2}(2)\right) \cong \mathbb{Z}_{2} \oplus \mathbb{Z}_{2}$, generated by

$$
\begin{aligned}
& \beta=\left[\widetilde{\mathrm{bI}}^{6}+\widetilde{\mathrm{bI}}^{7}+\widetilde{\mathrm{bI}}^{8}\right]=\left[\widetilde{\mathrm{bI}}^{2}+\widetilde{\mathrm{bI}}^{3}+\widetilde{\mathrm{bI}}^{4}+\widetilde{\mathrm{bI}}^{7}\right], \\
& \gamma=\left[\widetilde{\mathrm{bI}}_{o}^{2}+\widetilde{\mathrm{bI}}_{e}^{3}+\widetilde{\mathrm{bI}}_{e}^{4}+\widetilde{\mathrm{bI}}_{o}^{6}+\widetilde{\mathrm{bI}}_{e}^{8}\right]=\left[\widetilde{\mathrm{bI}}_{e}^{2}+\widetilde{\mathrm{bI}}_{o}^{3}+\widetilde{\mathrm{bI}}_{o}^{4}+\widetilde{\mathrm{bI}}_{e}^{6}+\widetilde{\mathrm{bI}}_{o}^{8}\right] .
\end{aligned}
$$

Note that the ranks of $C^{i}\left(b \mathcal{S}_{\mathrm{pr}}(3,2), \rho_{3,2}(2)\right), i=0,1,2$, are equal to 2,18 and 160 , respectively.

Let

$$
s_{\kappa *}: H^{\kappa}\left(b \mathcal{S}_{\mathrm{pr}}(3,2), \rho_{3,2}(2)\right) \rightarrow H^{\kappa}\left(b \mathcal{S}_{\mathrm{pr}}(2,1), \rho_{2,1}(2)\right)
$$

be the homomorphism induced by suspension $s_{\kappa}$. Note that $s_{\kappa}\left(\mathcal{F}_{*}\right)=\mathcal{F}_{*}$ for $\kappa=$ 0,1 and for each $\rho_{3,2}(2)$-class $\mathcal{F}_{*}$ of codimension $\kappa$ (see Remark 2.10). Note also that $s_{\kappa}=0$ for $\kappa=2$. Then, Lemma 3.5 shows that $s_{1 *} \beta$ induces a trivial $b \mathcal{S}_{\mathrm{pr}}$ cobordism invariant for stable Morse functions $f: V \rightarrow W$ on compact surfaces $V$ with boundary into $W=\mathbb{R}$ or $S^{1}$. Furthermore, by using the same method as in [7, Lemma 14.1], we can show that $s_{1 *} \gamma$ also induces a trivial $b \mathcal{S}_{\mathrm{pr}}$-cobordism invariant. Therefore, unfortunately, the above cohomology information does not provide us of a new cobordism invariant.

Let us now consider a certain restricted class of stable maps. For a positive integer $n$, let $\mathcal{A S}_{\mathrm{pr}}(n, n-1)$ be the set of fibers for proper admissible $C^{0}$ stable Thom maps of 
$n$-dimensional manifolds with boundary into $(n-1)$-dimensional manifolds without boundary, where a $C^{0}$ stable map $f: M \rightarrow N$ of an $n$-dimensional manifold with boundary into an (n-1)-dimensional manifold without boundary is admissible if it is a submersion on a neighborhood of $\partial M$. In other words, a stable map $f: M \rightarrow N$ of a 3-dimensional manifold with boundary into a surface without boundary is admissible if it has neither definite $\Sigma_{1,0}^{2,0}$ points nor indefinite $\Sigma_{1,0}^{2,0}$ points. Note that stable Morse functions on compact surfaces and their suspensions are always admissible.

Furthermore, set

$$
\mathcal{A} \mathcal{S}_{\mathrm{pr}}=\bigcup_{n=1}^{\infty} \mathcal{A} \mathcal{S}_{\mathrm{pr}}(n, n-1)
$$

This set, together with the equivalence relation induced by $\rho(2)$, which we still denote by $\rho(2)$ by abuse of notation, satisfies conditions (a)-(e) mentioned before.

Then a straightforward calculation shows the following.

Proposition 4.8 The cohomology groups of the universal complex

$$
\mathcal{C}\left(\mathcal{A S}_{\mathrm{pr}}(3,2), \rho_{3,2}(2)\right)
$$

for admissible stable maps of 3-manifolds with boundary to surfaces without boundary with respect to the $C^{0}$ equivalence modulo two regular fiber components are described as follows:

(1) $H^{0}\left(\mathcal{A S} \mathcal{S}_{\mathrm{pr}}(3,2), \rho_{3,2}(2)\right) \cong \mathbb{Z}_{2}$, generated by $\left[\widetilde{\mathrm{b}}_{o}+\widetilde{\mathrm{b} 0}_{e}\right]$.

(2) $H^{1}\left(\mathcal{A} \mathcal{S}_{\text {pr }}(3,2), \rho_{3,2}(2)\right) \cong \mathbb{Z}_{2} \oplus \mathbb{Z}_{2} \oplus \mathbb{Z}_{2}$, generated by

$$
\begin{aligned}
& \alpha=\left[\widetilde{\mathrm{bI}}^{2}+\widetilde{\mathrm{bI}}^{3}+\widetilde{\mathrm{bI}}^{4}+\widetilde{\mathrm{bI}}^{5}+\widetilde{\mathrm{bI}}^{9}+\widetilde{\mathrm{bI}}^{10}\right], \\
& \beta=\left[\widetilde{\mathrm{bI}}^{2}+\widetilde{\mathrm{bI}}^{3}+\widetilde{\mathrm{bI}}^{4}+\widetilde{\mathrm{bI}}^{7}\right]=\left[\widetilde{\mathrm{bI}}^{6}+\widetilde{\mathrm{bI}}^{7}+\widetilde{\mathrm{bI}}^{8}\right], \\
& \gamma=\left[\widetilde{\mathrm{bI}}_{o}^{2}+\widetilde{\mathrm{bI}}_{e}^{3}+\widetilde{\mathrm{bI}}_{e}^{4}+\widetilde{\mathrm{bI}}_{o}^{6}+\widetilde{\mathrm{bI}}_{e}^{8}\right]=\left[\widetilde{\mathrm{bI}}_{e}^{2}+\widetilde{\mathrm{bI}}_{o}^{3}+\widetilde{\mathrm{bI}}_{o}^{4}+\widetilde{\mathrm{bI}}_{e}^{6}+\widetilde{\mathrm{bI}}_{o}^{8}\right] .
\end{aligned}
$$

Note that the ranks of $C^{i}\left(\mathcal{A S}_{\mathrm{pr}}(3,2), \rho_{3,2}(2)\right), i=0,1,2$, are equal to 2,18 and 154 , respectively.

Let

$$
s_{\kappa *}: H^{\kappa}\left(\mathcal{A S}_{\mathrm{pr}}(3,2), \rho_{3,2}(2)\right) \rightarrow H^{\kappa}\left(\mathcal{A S}_{\mathrm{pr}}(2,1), \rho_{2,1}(2)\right)
$$

be the homomorphism induced by suspension $s_{\kappa}$. Then we obtain the following cobordism invariants. 


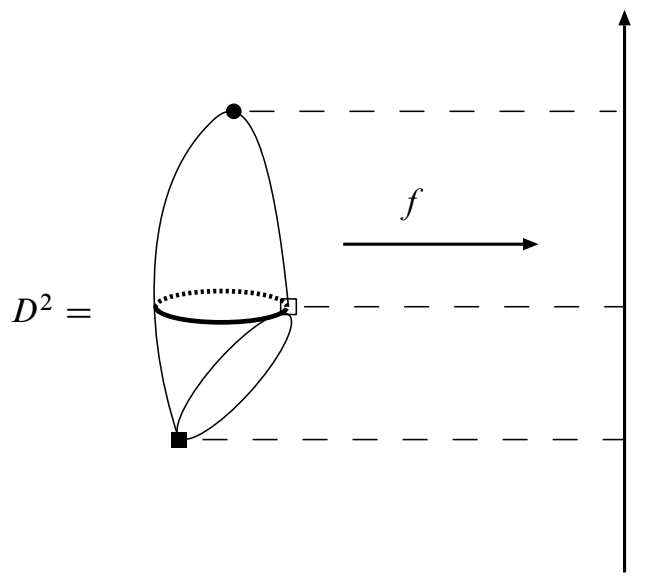

Figure 7: A stable Morse function on the disk

Corollary 4.9 Let $W$ be the real line $\mathbb{R}$ or the circle $S^{1}$.

(1) The cohomology class $s_{1 *} \alpha$ induces a nontrivial $\mathcal{A} \mathcal{S}_{\mathrm{pr}}$-cobordism invariant for stable Morse functions $f: V \rightarrow W$ on compact surfaces $V$ with boundary into $W$.

(2) The cohomology classes $s_{1 *} \beta$ and $s_{1 * \gamma}$ induce trivial $\mathcal{A} \mathcal{S}_{\text {pr }}$-cobordism invariants for stable Morse functions $f: V \rightarrow W$ of compact surfaces $V$ with boundary into $W$.

Proof A stable Morse function $f: D^{2} \rightarrow \mathbb{R}$ given by the height function as depicted in Figure 7 shows that the cobordism invariant $s_{1 *} \alpha$ is nontrivial, where $D^{2}$ denotes the 2-dimensional disk. The same construction works also for $W=S^{1}$.

On the other hand, Lemma 3.5 shows that the invariant associated with $s_{1 *} \beta$ is trivial. The triviality of the invariant associated with $s_{1 *} \gamma$ is proved by using the same argument as in [7, Lemma 14.1], so we omit the details here.

Remark 4.10 The above corollary shows that even if we take a nontrivial cohomology class of the universal complex, the corresponding cohomology class in the target manifold can be always trivial.

Remark 4.11 We can also consider co-orientable singular fibers in the sense of [7, Definition 10.5] and calculate the first cohomology group of the universal complex of co-orientable fibers for proper admissible stable maps of 3-dimensional manifolds with boundary into surfaces without boundary with respect to the $C^{0}$ equivalence modulo two regular fibers. Unfortunately, the cobordism invariants that we get are all trivial, so we omit the details here (for details, see [13]). 
Acknowledgements The authors would like to express their sincere gratitude to Shigeo Takahashi, Daisuke Sakurai, Hsiang-Yun Wu, Keisuke Kikuchi and Hamish Carr for stimulating discussions and for posing interesting questions. The authors would also like to thank the referee for very important comments which drastically improved the presentation of the paper. The first author has been supported in part by JSPS KAKENHI Grant Number 23244008, 23654028, 25540041, 15K13438. The second author has been supported in part by JSPS KAKENHI Grant Number 23654028, 15K13438.

\section{References}

[1] J Damon, The relation between $C^{\infty}$ and topological stability, Bol. Soc. Brasil. Mat. 8 (1977) 1-38 MR0649254

[2] C G Gibson, K Wirthmüller, A A du Plessis, E J N Looijenga, Topological stability of smooth mappings, Lecture Notes in Mathematics 552, Springer, Berlin (1976) MR0436203

[3] M Golubitsky, V Guillemin, Stable mappings and their singularities, Graduate Texts in Mathematics 14, Springer, New York (1973) MR0341518

[4] L F Martins, A C Nabarro, Projections of hypersurfaces in $\mathbb{R}^{4}$ with boundary to planes, Glasg. Math. J. 56 (2014) 149-167 MR3137856

[5] T Ohmoto, Vassiliev complex for contact classes of real smooth map-germs, Rep. Fac. Sci. Kagoshima Univ. Math. Phys. Chem. 27 (1994) 1-12 MR1341346

[6] A du Plessis, T Wall, The geometry of topological stability, London Mathematical Society Monographs, New Series 9, Clarendon Press, New York (1995) MR1408432

[7] O Saeki, Topology of singular fibers of differentiable maps, Lecture Notes in Mathematics 1854, Springer, Berlin (2004) MR2106689

[8] O Saeki, Cobordism of Morse functions on surfaces, the universal complex of singular fibers and their application to map germs, Algebr. Geom. Topol. 6 (2006) 539-572 MR2220688

[9] O Saeki, S Takahashi, Visual data mining based on differential topology: a survey, Pac. J. Math. Ind. 6 (2014) Article 4 MR3404141

[10] O Saeki, S Takahashi, D Sakurai, H-Y Wu, K Kikuchi, H Carr, D Duke, T Yamamoto, Visualizing multivariate data using singularity theory, from: "The impact of applications on mathematics", (M Wakayama, R S Anderssen, J Cheng, Y Fukumoto, R McKibbin, K Polthier, T Takagi, K-C Toh, editors), Math. Ind. (Tokyo) 1, Springer, Tokyo (2014) 51-65 MR3445542

[11] O Saeki, T Yamamoto, Singular fibers of stable maps and signatures of 4-manifolds, Geom. Topol. 10 (2006) 359-399 MR2224462 
[12] O Saeki, T Yamamoto, Singular fibers and characteristic classes, Topology Appl. 155 (2007) 112-120 MR2368204

[13] O Saeki, T Yamamoto, Co-orientable singular fibers of stable maps of 3-manifolds with boundary into surfaces, Sūrikaisekikenkyūsho Kōkyūroku 1948 (2015) 137-152

[14] N Shibata, On non-singular stable maps of 3-manifolds with boundary into the plane, Hiroshima Math. J. 30 (2000) 415-435 MR1799298

[15] V A Vassilyev, Lagrange and Legendre characteristic classes, Advanced Studies in Contemporary Mathematics 3, Gordon and Breach, New York (1988) MR1065996

[16] T Yamamoto, Classification of singular fibres of stable maps of 4-manifolds into 3-manifolds and its applications, J. Math. Soc. Japan 58 (2006) 721-742 MR2254408

[17] T Yamamoto, Euler number formulas in terms of singular fibers of stable maps, from: "Real and complex singularities", (A Harris, T Fukui, S Koike, editors), World Sci., Hackensack, NJ (2007) 427-457 MR2336698

[18] T Yamamoto, Singular fibers of two-colored maps and cobordism invariants, Pacific J. Math. 234 (2008) 379-398 MR2373454

Institute of Mathematics for Industry, Kyushu University

Motooka 744, Nishi-ku, Fukuoka 819-0395, Japan

Faculty of Engineering, Kyushu Sangyo University

3-1 Matsukadai 2-chome, Higashi-ku, Fukuoka 813-8503, Japan

saeki@imi.kyushu-u.ac.jp, yama.t@ip.kyusan-u.ac.jp

Received: 26 May 2014 Revised: 18 June 2015 